\title{
Exploring mobile health applications for self-management of Diabetes Mellitus
}

\author{
Ericles Andrei Bellei*, Hugo Roberto Kurtz Lisboa, Ana Carolina B. De Marchi
}

Dissertação defendida no Programa de Pós-Graduação em Computação Aplicada Instituto de Ciências Exatas e Geociências - Universidade de Passo Fundo

BR 285, São José, Passo Fundo - RS - Brasil

*168729@upf.br

\begin{abstract}
Resumo. Na rotina de tratamento de Diabetes Mellitus (DM), o paciente precisa considerar diversos dados e fatores. Nessa perspectiva, esta dissertação apresenta um aplicativo para acompanhamento da interdependência entre os fatores de tratamento de DM Tipo 1 com uma abordagem de visualização de dados interativa. Inicialmente, foi realizada uma revisão sistemática de literatura para investigar as funcionalidades e as técnicas de fundamentação dos aplicativos existentes. Na sequência, um aplicativo foi prototipado, avaliado preliminarmente com 76 pacientes, e implementado junto de um painel de controle web. Por fim, foi conduzido um experimento piloto com 4 pacientes e uma avaliação de satisfação com 97 pacientes e 9 profissionais de saúde.
\end{abstract}

\begin{abstract}
Patients with diabetes mellitus (DM) needs to deal with many data and consider diverse factors in the treatment routine. In this regard, this master's thesis presents an app for monitoring the linkage among treatment factors of Type 1 DM with an interactive data visualization approach. Initially, we systematically reviewed the literature to investigate DM app's features, the basis for its design and testing. Next, an app was prototyped, preliminarily assessed with 76 patients, and deployed along with a website dashboard. Afterward, we conducted a pilot trial with 4 patients, and a satisfaction assessment with 97 patients and 9 health professionals.
\end{abstract}

\section{Caracterização do problema e motivação}

Aplicativos m-Health (saúde móvel), têm desempenhado um papel central para treinamento, autogerenciamento, monitorização remota e coleta de dados de tratamento de doenças crônicas, como o Diabetes Mellitus (DM) [Debon et al. 2019]. No entanto, [Wu et al. 2017] argumentam que os aplicativos atuais podem não ser bons o suficiente para suportar o gerenciamento intensivo do DM Tipo 1, uma vez que eventos adversos, como hipoglicemia, estão sendo negligenciados e precisam ser melhor abordados em novos estudos. [Chomutare et al. 2011] afirmam que os recursos dos aplicativos para DM são individualmente distintos, mas têm o potencial de funcionar como uma ferramenta integrada de autogerenciamento. Como exemplo, os autores aventam a hipótese de que um paciente poderia registrar atividades físicas, refeições ou ingestão de carboidratos, e teria uma forma de visualização para compreender com mais facilidade como os fatores do tratamento se correlacionam e afetam a glicemia.

$\mathrm{Na}$ rotina de tratamento de DM Tipo 1, o paciente precisa, constantemente, considerar muitas variáveis para realizar ações, tomar decisões e ajustar suas tarefas. 
Por exemplo, a dosagem de insulina depende da hora do dia, da quantidade de comida ingerida, da glicemia momentânea, das características da atividade física, entre outros [American Diabetes Association 2018]. Como resultado, há uma dificuldade em orquestrar tarefas de autocuidado devido à grande quantidade e à diversidade de dados que surgem no tratamento diário de DM [Bellei et al. 2018].

Nesse cenário, é necessário aplicar técnicas de filtragem para extrair informações relevantes, além de oportunizar interfaces de usuário e métodos de visualização de dados adequados para auxiliar nas tarefas de tratamento [Gay and Leijdekkers 2015; Bui et al. 2007]. A visualização interativa de dados pode orientar a educação do paciente e o feedback personalizado sobre as tarefas de tratamento, levando à formulação de hipóteses que direcionam a uma melhor compreensão sobre os fatores de DM [Skrøvseth et al. 2015]. Da mesma forma, uma correlação visual de elementos gráficos pode ajudar a associar relações e padrões de causalidade entre os registros de saúde do paciente [Bui et al. 2007]. Complementarmente, a coleta de dados com feedback personalizado é uma forma de aprimorar a resolução de problemas de autogerenciamento de DM, que é uma habilidade essencial para o um controle adequado [Hill-Briggs and Gemmell 2007].

\section{Objetivos}

Este estudo teve como objetivo geral desenvolver e avaliar uma solução m-Health para a monitorização da interdependência entre os fatores de tratamento do DM Tipo 1 (glicemia, insulina, alimentação e atividade física) com uma abordagem de visualização de dados interativa. Ao longo do estudo, os objetivos específicos foram: realizar uma revisão sistemática da literatura para mapear o estado da arte; desenvolver um aplicativo móvel e uma versão complementar em website; avaliar a aceitação do aplicativo com os pacientes; avaliar a aceitação e o potencial da aplicação com profissionais de saúde.

\section{Método}

A sequência de etapas para a realização deste estudo foi baseada no IDEAS (Integrate, Design, Assess, and Share), um framework proposto por [Mummah et al. 2016] para o desenvolvimento de intervenções digitais mais efetivas.

\subsection{Etapa 1: Insights}

Inicialmente, foi conduzida uma revisão sistemática da literatura para investigar os recursos de aplicativos para DM, a base para seu projeto e como eles foram validados [Bellei et al. 2018]. Depois, foram investigados os recursos interativos e visuais de tecnologias relacionadas ao DM, como dispositivos de monitorização contínua de glicose e bombas de insulina. Depreendeu-se que os aplicativos com melhores resultados abrangiam, pelo menos, os principais fatores de tratamento (glicemia, insulina, alimentação, e atividade física) e que o envolvimento pacientes e profissionais de saúde no projeto de aplicativo culminava em melhores resultados. Entre os recursos dos aplicativos disponíveis, observou-se que o uso de insulina foi pouco abordado em forma de visualização gráfica. Além disso, menos da metade dos aplicativos usava os dados registrados para fazer recomendações ou prover feedback personalizado aos pacientes. Os dados levantados ensejaram a ideação dos recursos do aplicativo por uma equipe de especialistas, endocrinologista e paciente com DM. 


\subsection{Etapa 2: Design, feedback e implementação}

Com a plataforma Quant-UX, elaborou-se um protótipo de alta fidelidade, completo e interativo. Quant-UX também viabilizou testes com usuários remotamente. Para coletar feedback, foi desenvolvido um questionário on-line com instruções de acesso ao protótipo, perguntas sociodemográficas e sobre o design e funcionalidades do protótipo. $\mathrm{O}$ questionário foi divulgado em grupos de redes sociais de pacientes com DM. Após análise do feedback, o projeto do aplicativo foi reajustado e implementado. Um aplicativo Android foi desenvolvido com o framework React Native e todos os dados foram centralizados no banco de dados em nuvem Firebase. Com o Angular framework, foi implementado um painel de acompanhamento em um website para a exportação de relatórios e a monitorização remota em tempo real.

\subsection{Etapa 3: Avaliação}

Primeiro, um estudo piloto com 4 pacientes do projeto de extensão Acampamento da Criança do Diabetes verificou a integridade do aplicativo durante o uso por 1 semana e o método de avaliação por meio de um questionário embutido no próprio aplicativo e disparado remotamente. Depois, o aplicativo foi disponibilizado na Google Play Store e difundido com convites de instalação para pessoas em grupos de redes sociais de pacientes com DM Tipo 1. Incorporado ao aplicativo, um questionário de avaliação de satisfação foi configurado para aparecer ao paciente que usasse o aplicativo e completasse registros de no mínimo 1 dia de uso, assumindo-se a hipótese de que o paciente teria, então, noção suficiente para formar sua opinião. Foram consideradas as 97 avaliações registradas nas primeiras 4 semanas após o lançamento dos testes do aplicativo. Por fim, 9 profissionais de saúde com experiência em DM (4 médicos endocrinologistas, 4 nutricionistas e 1 enfermeira) avaliaram o aplicativo em relação a sua operação, recursos, benefícios, relevância e potencial.

\section{Resultados}

O aplicativo projetado e desenvolvido (Figura 1) permite o registro de dados das rotinas de glicemia, aplicação de insulina, ingestão de alimentos e atividade física, para gerar uma visualização gráfica, interativa e associativa dos dados, facilitando a identificação da interdependência entre os fatores de tratamento. Além disso, há um um algoritmo que fornece feedback personalizado sobre glicemia fora da faixa ideal, indicando suas possíveis causas e horário de ocorrência, verificadas conforme os registros efetuados. O aplicativo conta com um painel de visualização em um website para permitir a exportação de relatórios e a monitorização remota em tempo real por terceiros.

$\mathrm{Na}$ etapa de avaliação final, a média da concordância dos 97 pacientes foram: eu gostei dos recursos do aplicativo (87,5\%); eu gostei do leiaute, cores e elementos da interface do aplicativo (90,6\%); eu gostei dos gráficos e da apresentação de dados visuais neste aplicativo (94,2\%); minha experiência geral em usar este aplicativo foi satisfatória (81,5\%); eu acredito que este aplicativo possa facilitar meu tratamento diário com diabetes $(87,4 \%)$; eu pretendo continuar usando este aplicativo no meu dia-a-dia (91,9\%); eu recomendaria este aplicativo para outros pacientes $(95,4 \%)$. Em sua avaliação qualitativa, os 9 profissionais de saúde afirmaram que acreditavam que o aplicativo pode facilitar a prática clínica para acompanhar pacientes, que o recomendariam aos pacientes, que a forma de visualização em gráficos é útil e produtiva, e que o algoritmo de feedback personalizado pode detectar as falhas do tratamento, auxiliando em sua compreensão. 


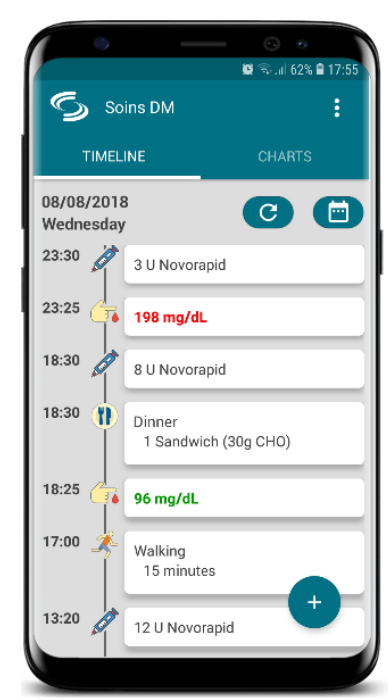

(a)

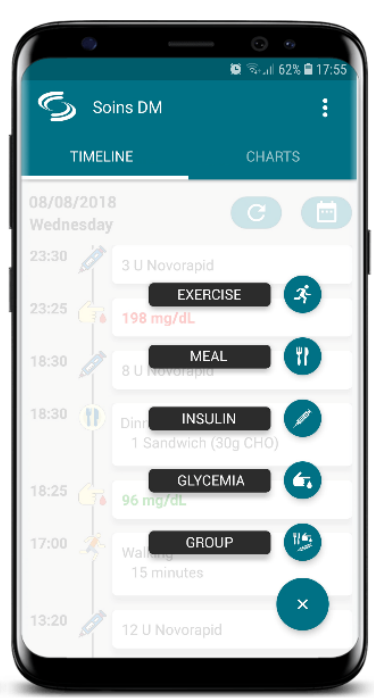

(b)

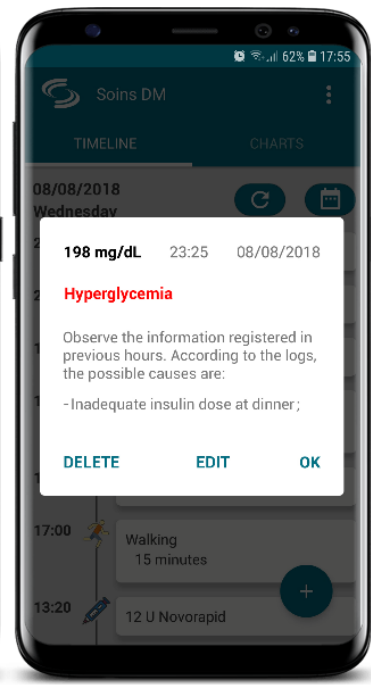

(c)

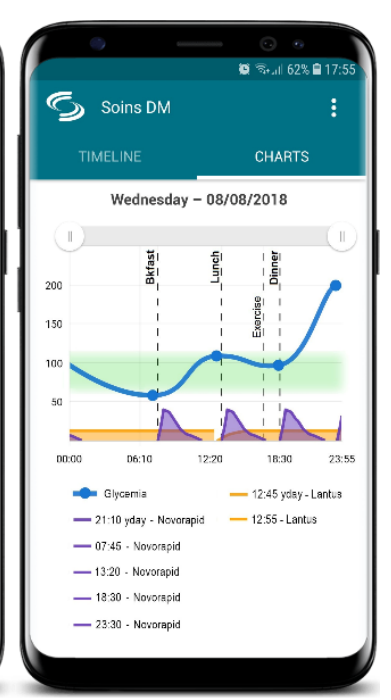

(d)

Figura 1. Capturas das principais telas do aplicativo: (a) visualização dos eventos de tratamento em formato de linha do tempo; (b) floating action menu para registro de novos dados; (c) feedback personalizado sobre uma glicemia fora do alvo com indicação das possíveis causas; (d) gráfico temporal interativo integrando variação glicêmica, eventos de alimentação, eventos de atividade física, duração e perfil farmacológico de ação da insulina utilizada pelo paciente.

\section{Discussão}

A literatura recomenda como essencial o envolvimento de pacientes e profissionais de saúde no desenvolvimento de software relacionado à saúde [Van Velthoven et al. 2018, Cechetti et al. 2019]. Este estudo foi uma iniciativa de um paciente e um endocrinologista, e recebeu colaboração e avaliação de outros pacientes e profissionais ao longo de seu desenvolvimento. A validação das premissas de design ocorreu em cada etapa do projeto. As avaliações on-line e incorporadas ao aplicativo aprimoraram o alcance e o acesso, facilitando os experimentos diretamente no contexto de uso real do paciente.

Estudos vêm demonstrado os efeitos, eficiência e benefícios de gráficos e visualização de dados para DM e seus correlatos [Wong et al. 2017]. O gráfico temporal interativo deste estudo permite inspeção e interpretação da interação, causalidade e interdependência entre os fatores de tratamento do DM1 e suas circunstâncias. Além disso, esse gráfico permite a noção dos efeitos da insulina ativa, em curso e ao longo das horas do dia [Hirsch 2005]. As abordagens de visualização têm um valor significativo, pois permitem encontrar informações ocultas nos dados, obter insights e evidências e tomar decisões mais bem informadas [Ko and Chang 2018].

O potencial e os benefícios do aplicativo ficaram evidentes com as avaliações. Há um consenso de que o aplicativo pode ser útil para pacientes e, especialmente, para profissionais também. As opiniões sobre os gráficos corroboram com a literatura quanto à sua utilidade para inferir eventos e padrões. $\mathrm{O}$ algoritmo de feedback personalizado foi visto como uma ferramenta proveitosa para o tratamento diário do DM, aprimorando o conhecimento do paciente, sua a capacidade em resolver problemas e seu controle glicêmico, uma vez que isso oportuniza a noção de causa e efeito [Mamykina et al. 2017]. Além disso, o aplicativo é uma ferramenta mais acessível que os métodos convencionais de monitorização contínua de glicose e tem recursos proporcionalmente vantajosos. 


\section{Conclusão}

Este trabalho explorou os recursos na literatura sobre aplicativos m-Health voltados a DM para desenvolver um novo aplicativo com visualização interativa e feedback personalizado, visando a facilitar a identificação da interdependência entre os fatores de tratamento de DM Tipo 1. O cenário de testes com pacientes e profissionais de saúde indica que o aplicativo desenvolvido é uma ferramenta útil, confiável e promissora. A abordagem de visualização e feedback foi o aspecto mais apreciado, considerada atrativa e vantajosa para a interpretação de informações do tratamento. Conclui-se que o aplicativo cumpre seu objetivo e pode auxiliar na monitorização de DM Tipo 1, reafirmando a tecnologia de aplicativos $\mathrm{m}$-Health como uma profícua ferramenta complementar de tratamento.

\section{Autoria, contribuições e produções derivadas}

Este estudo foi desenvolvido como dissertação de E.A.B. [Bellei 2019], orientado por A.C.B.M. e coorientado por H.R.K.L. Parte da visualização gráfica do aplicativo foi desenvolvida no trabalho de [Rodriguez et al. 2019], premiado no Concurso de Trabalhos de Iniciação Científica do SBCAS 2019. Como outras produções diretas, um artigo no periódico Telemedicine Journal and e-Health apresentou a revisão sistemática [Bellei et al. 2018], enquanto outro, no mesmo periódico [Bellei et al. 2020], apresentou o desenvolvimento e a avaliação do aplicativo m-Health. O certificado de registro de software foi concedido pelo Instituto Nacional da Propriedade Industrial (INPI) no processo BR512018001045-8. As produções secundárias incluem os artigos de [Cechetti et al. 2019] e [Debon et al. 2019].

\section{Agradecimentos}

Ao Acampamento da Criança com Diabetes pelo espaço e suporte. A Daiana Biduski, João Pedro Mazuco Rodriguez e Natália Pinto Cechetti pelo auxílio. Ao Conselho Nacional de Desenvolvimento Científico e Tecnológico (CNPq) pelas bolsas DTI-C e DT Nível 2. À Fundação Universidade de Passo Fundo pela bolsa parcial de mestrado.

\section{Referências}

American Diabetes Association (2018). 8. Pharmacologic Approaches to Glycemic Treatment: Standards of Medical Care in Diabetes-2018. Diabetes Care, v. 41, n. S1.

Bellei, E. A., Biduski, D., Cechetti, N. P. and De Marchi, A. C. B. (2018). Diabetes Mellitus m-Health Applications: A Systematic Review of Features and Fundamentals. Telemed J E Health, v. 24, n. 11.

Bellei, E. A., Biduski, D., Lisboa, H. R. K. and De Marchi, A. C. B. (2020). Development and Assessment of a Mobile Health Application for Monitoring the Linkage Among Treatment Factors of Type 1 Diabetes Mellitus. Telemed J E Health, v. 26, n. 2.

Bellei, E. A. (2019). Exploring mobile health applications for self-management of Diabetes Mellitus. Master's Thesis. University of Passo Fundo. Disponível em http://tede.upf.br/jspui/handle/tede/1668

Bui, A. A. T., Aberle, D. R. and Kangarloo, H. (2007). TimeLine: Visualizing Integrated Patient Records. IEEE Trans Inf Technol Biomed, v. 11, n. 4. 
Cechetti, N. P., Bellei, E. A., Biduski, D., et al. (2019). Developing and implementing a gamification method to improve user engagement: A case study with an m-Health application for hypertension monitoring. Telemat Informatics, v. 41.

Chomutare, T., Fernandez-Luque, L., Årsand, E. and Hartvigsen, G. (2011). Features of Mobile Diabetes Applications: Review of the Literature and Analysis of Current Applications Compared Against Evidence-Based Guidelines. J Med Internet Res, v. 13, n. 3 .

Debon, R., Coleone, J. D., Bellei, E. A., and De Marchi, A. C. B. (2019). Mobile health applications for chronic diseases: A systematic review of features for lifestyle improve-ment. Diabetes Metab Syndr Clin Res Rev, v. 13, n. 4.

Gay, V. and Leijdekkers, P. (2015). Bringing Health and Fitness Data Together for Connected Health Care: Mobile Apps as Enablers of Interoperability. J Med Internet Res, v. 17, n. 11.

Hill-Briggs, F. and Gemmell, L. (2007). Problem Solving in Diabetes Self-management and Control. Diabetes Educ, v. 33, n. 6.

Hirsch I.B. (2005) Insulin Analogues. N Engl J Med, v. 352 n. 2.

Ko, I. and Chang, H. (2018). Interactive data visualization based on conventional statistical findings for antihypertensive prescriptions using National Health Insurance claims data. Int J Med Inform, v. 116.

Mamykina, L., Heitkemper, E. M., et al. (2017). Personal discovery in diabetes selfmanagement: Discovering cause and effect using self-monitoring data. J Biomed Inform, v. 76.

Mummah, S. A., Robinson, T. N., King, A. C., Gardner, C. D. and Sutton, S. (2016). IDEAS (integrate, design, assess, and share): A framework and toolkit of strategiesfor the development of more effective digital interventions to change health behavior. J Med Internet Res, v.18, n. 12.

Rodriguez, J. P. M., De Marchi, A. C. B., Bellei, E. A., Biduski, D., and Lisboa, H.R. K. (2019). Uma abordagem de visualização gráfica para compreensão da relação entre os fatores do tratamento de diabetes mellitus tipo 1. In Anais Estendidos do XIX Simpósio Brasileiro de Computação Aplicada à Saúde (CTIC-SBCAS 2019).

Skrøvseth, S. O., Årsand, E., Godtliebsen, F. and Joakimsen, R. M. (2015). Data-Driven Personalized Feedback to Patients with Type 1 Diabetes: A Randomized Trial. Diabetes Technol Ther, v. 17, n. 7.

Van Velthoven, M. H., Wyatt, J. C., Meinert, E., Brindley, D. and Wells, G. (2018). How standards and user involvement can improve app quality: A lifecycle approach. Int $J$ Med Inform, v. 118, p. 54-57.

Wong, J. C., Neinstein, A. B., Look, H., et al. (2017). Pilot Study of a Novel Application for Data Visualization in Type 1 Diabetes. J Diabetes Sci Technol, v. 11, n. 4.

Wu, Y., Yao, X., Vespasiani, G., et al. (2017). Mobile App-Based Interventions to Support Diabetes Self-Management: A Systematic Review of Randomized Controlled Trials to Identify Functions Associated with Glycemic Efficacy. JMIR mHealth and uHealth, v. 5, n. 3 . 